Chapter 9

\title{
Old versus New - Tumor Ablation versus Tumor Nanoablation with Particular Emphasis on Liver Tumors
}

\author{
Zeno Sparchez, Tudor Mocan and Pompilia Radu \\ Additional information is available at the end of the chapter \\ http://dx.doi.org/10.5772/61008
}

\begin{abstract}
Loco-regional treatments play a key role in the management of hepatocellular carcinoma (HCC). Image-guided tumor ablation is recommended in patients with early-stage HCC when surgical options are precluded. Radiofrequency ablation is currently established as the standard method for local tumor treatment. Despite major advances in tumor ablation techniques the disease recurs in a high proportion of cases. A major limitation in its overall effectiveness is due to the difficulties of heating large tumors. Small regions of viable tumor may still remain even after apparently good tumor ablation by perfusion-mediated tissue cooling, preventing the whole tumor reaching a sufficient temperature for coagulation and necrosis. Moreover simple heating techniques have trouble discriminating between tumors and surrounding healthy tissues leading to many side effects. In order to overcome these major limitations numerous groups are investigating the use of energy-absorbing agents localized within tumor tissues to facilitate localized heating. A personal answer based on the review of the literature will be offered to the following questions: NIR photothermal therapy, RFA with nanoparticles, or magnetic fluid hyperthermia for the long term management of HCC? How should we deliver nanoparticles: systemically or directly intratumoral? Ablation versus mild hyperthermia: Pros and Cons in the majority of cases, hyperthermia is applied in one of two ways: a) high temperature for short time periods commonly referred to as ablation, or b) lower temperatures for long time periods, often called mild hyperthermia. The former is used to kill cells directly with heat and consequently can be used to thermally ablate tumor. The second method is just above physiological temperature, and these temperatures are more often used to trigger release from thermosensitive drug carriers. Both approaches can be combined with heat-sensitive drug targeting. There are many ways to induce nanoparticle mediated thermal therapy in solid tumors including absorption of infrared light, radiofrequency ablation and magnetically induced heating. These approaches have demonstrated high efficacy in preclinical models of HCC and are already tested in human clinical trials.
\end{abstract}

Keywords: Nanoparticles, liver tumors, percutaneous ablation 


\section{Introduction}

Hepatocellular carcinoma (HCC) occurs predominantly in patients with chronic liver disease and limited hepatic functional reserve. Therefore, surgical removal of HCC is feasible only in $15-20 \%$ of cases and non-surgical modalities play a relatively important role in HCC management. There are several non-surgical methods; however, ablation therapy has become a mainstay in particular for early-stage HCC because of its superb local control capability and high safety profile [1].

Ablation modalities currently available include percutaneous ethanol injection (PEI), radiofrequency ablation (RFA), microwave ablation (MWA), cryoablation, laser ablation (LA), and irreversible electroporation.

PEI was one of the first effective ablative techniques to be widely adopted for the treatment of small HCC. Ethanol causes dehydration and subsequently necrosis [2]. As far as PEIs are concerned, the 5-year survival rates in patients with HCCs measuring less than $3 \mathrm{~cm}$ range from $47 \%$ to $65 \%$ and in a recent study of 685 Japanese patients, the $5-, 10-$, and 20 -year survival rates $-49 \%, 18 \%$, and $7.2 \%$, respectively, were similar to those observed in patients with cirrhosis who did not have HCC [3]. PEI maintains the advantage of allowing the treatment of tumors near sensitive organs and tissues; however the applicability of PEI in larger HCC has been shown to produce incomplete necrosis mainly due to the heterogeneous consistency of these tumors [4]. Moreover, PEI is of little benefit in infiltrating HCC or in metastases.

Current limitations of PEI can be overcome with RFA. Radiofrequency current induces ionic agitation that in turn results in heating. The superiority of RFA to PEI in prolonging patient survival has been shown in a randomized controlled trial [5]. The 3-year survival rates were $48 \%-67 \%$ following PEI and $63 \%-81 \%$ following RFA. Moreover, Chen et al. performed a randomized control trial between RFA and hepatectomy in patients who had HCC $\leq 5 \mathrm{~cm}$ and found the same overall and recurrence-free survival between the two patient groups [6]. A major disadvantage of RFA is mainly the difficulty to target HCC located in "problem" areas of the liver, for instance tumors adjacent to blood vessels, settings in which the diffusion of heat is less advisable [7]. This phenomenon is also known as the heat-sink effect.

In the last two years, MWA has gained acceptance as a favorable alternative and in some cases a preferred choice of ablation alternative. In MWA, the mechanism of heat generation is based on rapid frictional movement of water molecules in high-frequency (900-2500 MHz) electromagnetic field. The tissue's polar molecules are forced to continuously realign with the oscillating electric field, increasing their kinetic energy, and hence the temperature of the tissue [8]. Unlike RFA, microwaves are capable of effectively heating and propagating through many types of tissue, even those with low electrical conductivity, high impedance, or low thermal conductivity. Moreover, they can readily penetrate through the charred or desiccated tissues that tend to build up around all hyperthermic ablation applicators, resulting in limited power delivery for non-microwave energy systems [9].

MWA has several theoretical advantages, including greater penetration of energy into tissues resulting in a larger area of ablation, higher intratumoral temperatures, faster ablation times, 
less susceptibility to the heat-sink effect, no need for grounding pads, and low sensitivity to local variation in tissue physiological properties [10]. In some studies, MWA has been compared with RFA for the treatment of HCCs of different sizes $(<3 \mathrm{~cm}$ and $<5 \mathrm{~cm})$ and despite the theoretical advantages of MWA, no significant differences have been observed in either setting with regard to the completeness of tumor necrosis, disease recurrence, survival, or complication rates $[11,12]$.

Laser thermal ablation is another technique that has been associated with high rates of complete necrosis (an average of 95\%) in HCCs measuring less than $3 \mathrm{~cm}$ [13]. Unfortunately, there are only a few centers that use this type of ablation and therefore the amount of data is limited. Moreover, it is based on sophisticated technology, requires much more substantial operator experience, and involves placement of multiple optical fibers within the neoplastic lesion according to a programmed spatial distribution scheme [14]. Although more expensive to set up and support than RF, LAs are a little more predictable.

To date, there are only a few studies comparing LA with RFA in hepatocellular carcinoma. In their randomized controlled prospective study, Ferrary et al. [15] treated 81 cirrhotic patients with 95 biopsy proven $\leq 4 \mathrm{~cm}$ HCCs comparing LA with RF ablation. Two matched groups were randomized to US-guided RF or LA under general anesthesia. The authors adopted multiple fiber techniques using $5 \mathrm{~W}$ per fiber delivering a maximum of $1800 \mathrm{~J}$ per fiber per single illumination. They reported no significant overall differences in survival rates between the two methods with cumulative rates of $91.8 \%, 59 \%$, and $28.4 \%$ at 1,3 , and 5 years, respectively. However, they demonstrated a statistically significant higher survival rate for RF over LA for Child A patients $(\mathrm{p}=0.9966)$ and nodules $\leq 2.5 \mathrm{~cm}(\mathrm{p}=0.01181)$. In a randomized prospective trial in a single center with three years of follow-up, the authors treated 140 patients with 157 biopsy-proven HCCs to compare LA and RFA (70 patients with 77 nodules and 70 patients with 80 nodules, respectively). Median follow-up in RFA and LA groups was 21 and 22.5 months, respectively. Complete response was observed in $97.2 \%$ and in $95.8 \%$ of RFA and LA group patients, respectively. Median time to tumor recurrence was 25.6 and 37.8 months in RFA and in LA groups, respectively $(\mathrm{P}=0.129)$. Estimated probability of survival at 1,2 , and 3 years was $94 \%, 88 \%$, and $66 \%$ in the RFA group and $94 \%, 81 \%$, and $59 \%$ in the LA group, respectively $(\mathrm{p}=0.693)$. No major complications or significant treatment-related morbidity were observed in both groups. The authors concluded that LA was non-inferior to RFA either in obtaining the complete ablation of HCC nodules or in the long-term outcome [16].

Another type of percutaneous tumor ablation is represented by cryoablation (CRYO). Percutaneous CRYO is a promising local ablation technique, which is believed to ablate cancer cells by several mechanisms including intracellular ice formation, solute-solvent shifts that cause cell dehydration and rupture, and small-vessel obliteration with resulting hypoxia. Perhaps, the main advantage of CRYO relative to RFA is its precise intraprocedural monitoring of iceball formation via various imaging techniques [17]. There are a few studies comparing CRYO with other types of tumor ablation techniques; however Wang $C$ et al. report the results of a randomized, controlled multicenter trial comparing percutaneous CRYO and RFA in patients with cirrhosis, Child-Pugh class A or B liver function, and 1-2 HCC nodules measuring $\leq 4 \mathrm{~cm}$. The primary endpoints were local tumor progression at 3 years and safety. As for the former, 
CRYO proved to be significantly superior to RFA in patients with larger tumors (i.e., those that were 3.1 to $4 \mathrm{~cm}$ in diameter). The two methods were not significantly different in terms of complication rates, which were less than $4 \%$ in both groups, or survival (overall and tumorfree) at 1, 3, and 5 years [18]. The superiority of CRYO over RFA in the larger tumors suggests that CRYO has the ability to necrotize larger volumes of tissue, hence increasing the chances of ablating microsatellite lesions that are always possible with lesions of this size.

Irreversible electroporation (IRE) is a new treatment method with certain advantages over the existing ablative techniques that have gained widespread attention. With IRE, cell death is induced with electric energy. Under image guidance electrodes are placed around the tumor and through multiple and short high-voltage electric pulses, the existing cell membrane potential is disturbed. As a consequence, nanoscale defects appear in the lipid bilayer of the cell membrane. Although IRE is believed to destroy all cells within the ablation zone effectively, the non-thermal nature of IRE results in relative preservation of the extracellular matrix. Hence, the structural integrity of vessels and bile ducts remain intact. Moreover, IRE is not affected by the heat-sink effect [19]. All these advantages suggest that IRE may be more suitable for the treatment of HCCs ineligible for surgical resections or thermal ablation because of unfavorable location.

Currently, there are no published clinical trials for the treatment of hepatic tumors using IRE. In a recent review, Scheffer J. et al. included 221 patients with 325 lesions in different organs: 227 hepatic tumors, 70 unresectable pancreatic adenocarcinoma, 17 renal tumors, 8 pulmonary tumors, 1 presacral tumor, and 2 lymph nodes. Most of the patients were treated by IRE owing to tumor proximity to bile ducts, bronchi, renal pelvis, presacral neural plexus or large vessels, making the tumor unsuitable for surgery or thermal ablation. They concluded that IRE is a safe procedure with a promising early efficacy on smaller hepatic tumors near vascular structures and portal triads, with reported ablation success reaching $90 \%$, but rapidly decreasing with increasing tumor size [20].

Tremendous efforts have been made in the last decades to improve the currently available techniques. However, given that there is not a single method available that meets all the requirements of an ideal ablation system, based on what has been discussed above and on data from the vast literature available, we can reasonably draw some conclusions.

Firstly, all differences between the techniques in terms of results are modest. Secondly, one technique may be more difficult than another and more rapid than another. Thirdly, each technique has its own major advantages and disadvantages. Finally, the rate of recurrence is still high after tumor ablation despite the major advances in tumor ablation devices, optic fibers, and improved imaging guidance. A major limitation in its overall effectiveness is due to the difficulties of heating large tumors. Small regions of viable tumor may still remain even after apparently good tumor ablation. Moreover, simple heating techniques have trouble discriminating between tumors and surrounding healthy tissues leading to many side effects. In order to overcome these major limitations, numerous groups are investigating the use of different types of nanoparticles, including carbon nanotubes, gold nanoparticles, and magnetic nanoparticles, placed/ introduced within tumor tissues to facilitate localized heating. 


\section{Molecular mechanism of nanoparticle-mediated tumor ablation}

A better understanding of the molecular mechanism of nanoparticle mediated tumor ablation is of great importance in order to improve the current available ablation techniques and also to increase the synergies between specific drugs and tumor ablation. There are several ways in which nanoparticles (NPs) alone can affect biological processes.

Several studies have shown that NPs can increase the production of reactive oxygen species (ROS). Cancer cells are generally deficient in antioxidative enzymes present in normal cells, making them more vulnerable to an oxidative assault. Iron oxide nanoparticles via direct uptake in cancer cells result in acutely elevated intracellular iron concentrations and subsequent ROS generation by Fenton reaction [21]. Moreover, silver nanoparticles have also been linked to ROS generation via a mechanism affecting calcium homeostasis. Silver ions can act on the same sites as calcium ions that could perturbate calcium influx in and out of the mitochondria. As a consequence, mitochondrial membrane damage results in ROS production, inhibition of ATP synthesis, and initiation of apoptotic signaling pathways [22].

From a biological and molecular point of view, NPs can affect different structures of the cancer cells. For instance, cellular uptake of NPs results in changes to the cytoskeleton and further affects many biological processes including cell spreading and adhesion, cell growth, viability, and ECM production [23]. Moreover, the accumulation of NPs in the cytoplasm may lead to physical interactions with the cytoskeleton, an increase in size and/or number of endosomes leading to the rearrangement of the cytoskeleton components in order to form new trafficking routes [24]. We consider that by altering the intracellular trafficking routes many other fundamental processes, including intracellular signaling pathways, different types of crosstalks with other cells and proliferation may also be affected. Furthermore, NPs can be engineered to accumulate preferentially in the nucleus of cancer cells. One study used gold nanoparticles (AuNPs) coated with polyethylene glycocol, bioconjugated with an argininegyicine-aspartic acid peptide and a nuclear localization signal peptide in order to transport the nanoparticles into the cancer cell nucleus. The results showed that nuclear targeting of AuNPs in cancer cells cause cytokinesis arrest, leading to the failure of complete cell division and thereby resulting in apoptosis [25].

In the past, cancer was considered an isolated self-sufficient ball of aberrant cells. However nowadays, tumors are viewed as "organs" composed of multiple and highly interactive cell types. Thus, the tumor is made up of primary cancer cells and of a court of stromal cells including mesenchymal derived cells, inflammatory cells, and vascular cells. Each of these cell types can be found in normal stroma, but in a tumorigenic setting, the cancer has appropriated, modified, and corrupted these cells to do its bidding [26]. NPs can also be used to target the tumor stroma changing the tumor microenvironment from its pro-tumorigenic state to an antitumorigenic state. One study demonstrated the ability of nanoparticles to target the tumor endothelium and improve the anti-tumoral efficacy of paclitaxel, both in vivo and in vitro [27]. Another approach would be to target the macrophage because they are inherently phagocytic and may uptake nanoparticles either within the tumor or in the circulation and subsequently migrate towards to the tumor. Another ability of macrophage is to store iron; hence, iron oxide 
NPs have been shown to induce cytotoxic effects on themselves and surrounding cells via ROSmediated activation of the c-jun N-terminal kinase pathway [28].

Understanding how nanomaterials affect live cell function, controlling such effects, and using them in therapy (for example In tumor ablation), is now the most challenging aspects of nanobiotechnology. An ideal NP would be a multifunctional one, targeting both the tumor cells and tumor microenvironment with low toxicity, which is easy to engineer, and has low costs. However, there is still a long way and a great deal of research has to be performed in order to develop what we consider the ideal nanoparticle.

\section{Near-infrared photothermal ablation}

Near-infrared (NIR) laser light is ideal for in vivo hyperthermia applications because of its low absorption by tissue chromophores such as hemoglobin and water. NIR light demonstrates maximal penetration of tissue, thereby reaching deep inside the tissue. Photothermal ablation (PTA) therapy is a recently developed technique that uses NIR laser light-generated heat to destroy tumor cells. In recent years, PTA has gained a lot of popularity mainly because a specific amount of photoenergy is delivered directly into the tumor without causing systemic effects [29]. However, this therapy approach is limited by the fact that the heating is nonspecific and nonuniform mainly in areas peripheral to large blood vessels where heat can be rapidly dissipated by circulating blood.

The efficacy of PTA can be significantly enhanced by using different types of nanoparticles that are applied to the target tissue to mediate selective photothermal effects. For instance, AuNPs including gold nanorods, gold nanocages, gold nanostars, and gold nanopopcorns with unique optical proprieties have been developed [30].

In order to treat a tumor, AuNPs are systemically administered to the subject and allowed to passively localize in the tumor. The tumor is then exposed to an excitation source such as the NIR laser light. The AuNPs absorb the incident energy and convert it into heat, which raises the temperature of the tissue and ablates the cancerous cells by disrupting the cell membrane [31]. AuNPs have unique optical-electronic proprieties as a result of surface plasmon resonances (SPRs). SPR is a phenomenon in which free electrons oscillate collectively at the interface of metal and surrounding medium in resonance with external electromagnetic fields [30].

Nanoparticles in the tissues produce heat strong enough for thermal ablation in both tumors and surrounding cells. Therefore, it is crucial to increase the intratumoral localization of the nanoparticles on the one hand and to protect the surrounding tissue on the other hand. Selective accumulation of AuNPs in the target tumor tissue can be achieved by surface conjugation of targeting agents, such as antibodies and peptides that can recognize specific cell types. For instance Liu et al. reported that gold nanoshells functionalized with the small peptide A54 can significantly increase the efficiency of cancer cell death in the NIR photothermal treatment due to the specific binding (targeting) between the A54-nanoshells and the liver cancer cells, BEL-7404 and BEL-7402 [32]. 
AuNP can also be functionalized to load various cargoes such as different types of anticancer drugs. As an example in this setting, You et al. investigated DOX-loaded hollow gold nanospheres (DOX@HAuNSs) and conjugated them with a peptide sequence that targets EPHB4, a tyrosine kinase receptor that is often overexpressed in many tumor cell membranes including HCC. NIR laser irradiation after treatment with targeted DOX@HAuNSs resulted in significantly suppressed tumor growth when compared with the control treatment with nontargeted DOX@HAuNSs or HAuNSs [33]. Moreover, another study conducted by the same authors, evaluated the triggered release of paclitaxel via NIR laser irradiation and its antitumor efficacy by hepatic arterial administration of HAuNS and paclitaxel loaded microspheres into rabbits with liver carcinoma in situ [34]. The results showed statistically significant increases in necrosis and apoptosis percentage in the MS-HAuNS-PTX-plus-NIR treatment group compared with the other two treatment groups.

A different approach in the field of NPs, mediated NIR thermal ablation has been developed in the last two years mainly due to the development of therenostic agents, which combine diagnostic and therapeutic modalities. This approach offers tremendous potential for the management of chronic liver injury or HCC. In a recent article, multifunctional nanoprobe based on Glypican-3 anti-body-mediated HCC-targeting Prussian blue nanoparticles (antiGPR-PBNPs) was developed as a novel theranostic agent for the targeted PTT and MR imaging of HCC treatments [35]. They concluded that antiGPC3-PBNPs could be used as a promising nanoprobe for further treating and early diagnosis of HCC.

A major limitation of nanoparticle-assisted drug delivery is represented by their uptake in the reticuloendothelial system leading to undesirable systemic toxicity and reduced efficacy. Hence many researchers have investigated the use of different cell types for drug delivery. Zhao J et al. in their study used adipose-derived mesenchymal cells (AD-MSCs) to deliver superparamagnetic iron oxide (SPIO)-loaded gold nanoparticles (SPIO@AuNP) into HCC tumors [36]. They demonstrated that AD-MSC is an effective carrier for the specific delivery of theranostic agents to liver injuries or HCC and SPIO@AuNP is a host-compatible cargo that enables both MRI enhancement and laser induced thermal ablation.

Besides the different types of gold nanoparticles described above, carbon nanotubes (CNT) also have the ability to efficiently convert NIR into heat. The role on CNT-mediated thermal therapy for the treatment of a wide variety of cancer types both in vitro and in vivo have been recently reviewed [37]. It is hard to claim that CNTs are better than GNPs because direct comparisons are hard to make; however, some estimates indicate that CNTs can achieve thermal destruction of tumors at 10-fold-lower doses and a 3-fold-lower power than what is required for gold nanorods [23]. On the other hand GNP can be synthesized with great uniformity and have already been tested in human clinical trials.

It is worth to mentioning that there is a massive amount of research in the field of nanoparticlesmediated PTA therapy. We only provided a few examples that we considered most suitable. Describing all the possible applications of nanoparticles mediated thermal therapy is beyond the purpose of this chapter. 


\section{Magnetic fluid hyperthermia-iron magnetic nanoparticles}

Thermotherapy represents a physical treatment induced by hyperthermia. Nowadays, macroscopic thermotherapy (ablative methods: microwave or radiofrequency, optical laser irradiation via fibers, focused ultrasound) is widely used to destroy focal tumors. The mechanism of tumoral damage is the result of an irreparable destruction of molecular constituents of cells (mainly protein denaturation) that appears after an exposure of a few minutes at temperatures higher than $48^{\circ} \mathrm{C}$. Even if it has lower side effects when compared to conventional therapy (chemo/radiotherapy) and although it has proved to be a reliable alternative to surgery, this therapy has several limits: the relative higher rate of incomplete destruction for tumors larger than $3 \mathrm{~cm}$ and a higher risk of destruction of the proximate healthy tissue. These deficiencies seem to disappear by using a new thermal method known as magnetic termic hyperthermia [38]. This approach uses an external alternating magnetic field applied to a target tumor where magnetic metallic particles (MNPs) have been infiltrated or injected. MNPs show distinguishing phenomena such as superparamagnetism, high field irreversibility, high saturation field, extra anisotropy contributions, or shifted loops after field cooling [39]. According to Reference [40], the distinguished phenomena noticed in MNPs are the result of the interaction between the intrinsic properties (size, distribution, and finite-size effects) and the interparticle interactions. The MNPs have the ability to absorb the energy of the alternating magnetic field energy and transform it into heat. Two factors are implicated in producing hyperthermia, the size of the magnetic material and the strength of the applied magnetic field. Larger implants (seeds) generate heat by resistance to circumferential eddy currents induced on the surface of the seeds by an alternative magnetic field [41]. Multidomain particles produce heat by hysteresis loss effects. On the contrary, nanoparticle, particularly subdomain particle, suspensions generate heat mainly by Brownian relaxation (heat is the result of friction arising from the total particle oscillations) and Neel relaxation (heat is the result of friction arising from the rotation of the magnetic moment with each field oscillation) [42, 43].

Superparamagnetic particles are particles that have sufficient high thermal motion after the magnetic field is removed, which can be randomly reoriented so as not to leave a residual magnetization [43].

Due to their properties, these particles may have several applications in clinical practice such as hyperthermia (HT), drug delivery and diagnosis (s.a nuclear magnetic resonance imaging).

HT represents a therapeutic procedure used to destroy a tumoral tissue at temperatures over $43^{\circ} \mathrm{C}$ [38]. It has been observed that tumoral cells have an increased thermal sensitivity in comparison to healthy cells; this feature is the result of an increased metabolism [44, 45]. Apoptosis is the result of cytotoxic effects that depend on physiological cell parameters (hypoxia or acidity) at temperatures over $43^{\circ} \mathrm{C} .43^{\circ} \mathrm{C}$ is the temperature limit over which the expression of HSPs is stimulated, which leads to antitumor immunity and apoptosis [46]. The antitumor immunity increases as a result of an enhanced presentation of tumoral antigenic peptide to a major histocompatibility complex (MHC). HSP70 expression reaches its maximum $24 \mathrm{~h}$ after heating. The increased MHC class I surface expression is slower, so it starts $24 \mathrm{~h}$ after applied hyperthermia and the peak is after $48 \mathrm{~h}$ [38]. Two mechanisms have been suggested. 
One of the possible mechanisms is that the heat induces the enhancement of antigenic peptide presentation through MHC class I antigens of tumor cells. Another possible mechanism is the cross-presentation of antigenic peptides by dedicated antigen-presenting cells (APCs) [46].

The advantage of magnetic hyperthermia is that it restricts the heating to the tumoral area, which presents both grand opportunities and challenges for the non-invasive treatment of tumors. Therefore, by combining this characteristic of the tumoral tissue with the MNPs property, it is obvious that the administration of MNPs (with the purpose of delivering toxic amounts of thermal energy to the tumoral tissue) will produce a more effective destruction of the tumoral tissue.

For clinical practice, MNPs must meet several criteria: they must be small enough to remain in the circulation after injection and pass through the capillary; they must not be an embolic agent; they must be non-toxic and non-immunogenic; they must maintain the initial structure; and they must be biodegradable. Another important property of these particles is to be highly magnetized in order for their movement to be controlled with a magnetic field so that they can be immobilized near the targeted tumoral area [47]. The most important factors, which determine the biocompatibility and toxicity of these materials, are the nature of the magnetically responsive component, the final size of the particles, their core, and their coatings [39]. The most utilized MNPs are magnetite (Fe3O4) or its oxidized form, maghemite ( $\gamma$-Fe2O3). Magnetite is easier to obtain than maghemite; therefore, most of the studies utilized magnetite [38]. In order to avoid the constitution of large aggregates, the modification from the original form and biodegradation, the MNPs are coated with a biocompatible polymer during or after the synthesis [39]. The particles' size influence the stability, tissular diffusion, effective surface areas (easier attachment of ligands), and the power of absorption at tolerable altering current magnetic fields. Therefore, only subdomain magnetic particles (nanometer-sized), especially particles smaller than $100 \mathrm{~nm}$ (so-called nanoparticles), can be utilized [48, 49]. Also, it is important to highlight that the heating potential is dependent on particle size and shape, and thus the use of uniform particles is essential for a rigorous control in temperature [39]. Therefore, the magnetic particles used may modify the energy, absorption rate, mode of energy deposition, application, and focusing. For this technique, the sizes of the particles are as follows: seeds (rods of several millimeter size), multidomain particles (1-300 mm), nanoparticles (1-100 nm), and subdomain particles (below $20 \mathrm{~nm}$ ) [41].

Gilchrist was the first author that showed promising results obtained after selective heating that followed the direct injection of a suspension of magnetic particles into draining lymph nodes from colon cancer [50]. In 2001, Moroz showed that hepatic arterial infusion of lipiodol containing ferromagnetic particles could result in an excellent targeting of liver tumors with hyperthermia on the subsequent application of an external alternating magnetic field [51]. The following years, encouraged by the results of the use of MNPs in animal studies (on mouse mammary carcinoma, glioblastoma, and prostate cancer), some authors focused on the improvement of HT techniques for clinical applications [52-56]. For in vivo delivery, the authors used thermosensitive liposomes, direct injection into the tumor, or the intravenous route. 
An important progress has been made in improving the quality of the MNPs; therefore, for construction, high temperature crystallization or different coatings were used, such as dextran, polyethylene glycol (PEG), dopamine, silanes and gold [43].

Several authors introduced MNPs either in the core or in between the lipid bilayer of thermosensitive liposomes and, on alternating magnetic field AMF heating, the encapsulated drugs were released [43]. Shinkai utilized liposomes where he introduced magnetite nanoparticles (with a diameter of $10 \mathrm{~nm}$ ). After administration, these nanoparticles increased the temperature of the tissue [57]. In another study, Ito injected magnetite cationic liposomes (MCLs) into the tumor tissue. They heated the tissue above $43^{\circ} \mathrm{C}$ and obtained a complete regression of mammary carcinomas in all mice [58]. Also, Jimbow [52] developed a particle with N-propionylcysteaminylphenol (NPrCAP) conjugated onto the surface of magnetite nanoparticles (NPrCAP/M). The result was the inhibition of melanoma cells growth as a result of the production of cytotoxic free radicals. In another study, a thermosensitive polymer was layered onto MNPs covalently coupled to doxorubicin with an acid-labile hydrazine bond that showed release on heating with AMF and a $\mathrm{pH}$ of 5.3 (the $\mathrm{pH}$ of endosomes) [59]. The authors combined via emulsification MNPs with a polyvinyl alcohol polymer and encapsulated hydrophobic/ hydrophilic drugs. The drugs were released after the heating with an alternative magnetic field [60].

Direct intratumoral injection was used in the first MNP HT clinical trial treating a patient with a recurrent prostatic tumor [61]. Through the use of transrectal ultrasound and fluoroscopy guidance, the authors performed a transperineal injection of the MNPs into the prostate. After the administration of MNPs, the particles were selectively heated in an externally applied alternative magnetic field. The conclusions of these trials were encouraging. Due to the low clearance of MNPs from tumors, serial heat treatments were possible after a single magnetic fluid injection. Another positive aspect was the fact that a low magnetic field was used to produce the necessary temperatures. Furthermore, this treatment does not cause discomfort or serious side effects. In these studies, the CT exam had an accuracy rate of $85 \%$ in evaluating the treatment-related parameters. The same good results were obtained later in human glioma trials $[62,63]$.

In 2008, Takamatsu et al. combined the intra-arterial selective HT with the transcatheter arterial embolization technique in a rabbit model for renal carcinoma [64]. For injection, they utilized a mixture of commercially available nano-sized magnetic particles (Ferucarbotran) and lipiodol as embolic material. The mixture was injected into the renal artery under fluoroscopic guidance. The intratumoral temperatures of $45^{\circ} \mathrm{C}$ were obtained after the area was exposed to an external alternating-current magnetic field. Even the result was not spectacular (the treated tumor was hypovascular) the authors speculated that this method can be used only in hypervascular tumors. In another study, Huang HS injected IV MNPs (1.9 mg Fe/g tumor) in a subcutaneous squamous cell carcinoma mouse model. After the injection, they applied a field of $38 \mathrm{kA} / \mathrm{m}$ at $980 \mathrm{kHz}$; therefore, the tumors could be heated to $60^{\circ} \mathrm{C}$ in $2 \mathrm{~min}$. The results were encouraging, showing an ablating with millimeter $(\mathrm{mm})$ precision and a surrounding tissue intact [43]. 
Intravenous administration has several advantages compared to sowing such as: it assures a more precise cover even for an irregular tumor and small tumors; it can be used for the treatment of metastasis (after one injection more than one tumor can be treated simultaneously); the distribution is more overall (rather than the dotted distribution from sowing); and it is minimally invasive $[43,48]$.

The evaluation of the iron concentrations can be mapped with high accuracy by MRI, computed tomography or magnetorelaxometry $[43,65,66]$.

The science of MNPs is still in its early stages. The recent results of magnetic HT in cancer therapy are very encouraging; but it is necessary to traverse the experimental stages into clinical practice to see the real applicability of this new technique.

\section{NP-based thermal therapy using radiofrequency}

\subsection{Standard RF-mediated nanoablation}

Standard RFA is an invasive procedure that requires the insertion of electrodes within the tumor. Tumor destruction occurs as a result of vibrations of ions within tumor tissue induced by radio waves, which give rise to friction and lethal heat. Although it is possible to achieve local control in liver tumors $<2.5 \mathrm{~cm}$, in larger lesions local tumor recurrence is common $[67,68]$.

Initially, in order to increase the efficacy of RFA, the ablation guidance methods were improved (contrast-enhanced ultrasound, fusion imaging, etc.), but this led only to a slight efficacy improvement. Because of the changes that occur after RFA (increased vascular and cellular membrane permeability), the periphery of the tumor becomes more susceptible to chemotherapy. Thus, the combination of thermal ablation and chemotherapy seemed to lead to promising results. The results of these methods did increase the efficacy of RFA, but it was not enough. Therefore, new treatments that will augment cytotoxicity at the margin of the ablation zone have been developed.

The efficiency of RFA can be significantly enhanced by administration of special thermal absorbing agents such as NPs, which are targeted into a tumor area (actively or passively) with the purpose to release locally the retained heat and thus enhance tumoral destruction.

The NPs in free form or those containing various anti-cancer agents may be administrated before, at the time, or after RFA [68, 69]. Administering CYT-6091, a TNF-labeled NP, $4 \mathrm{~h}$ prior to RFA yielded a significantly larger zone of central necrosis and a $23 \%$ increase in ablation volume in comparison to RFA alone [69]. Using this NP enhanced ablation, the partially ablated tissue at the periphery was replaced by completely ablated tissue [69].

The administration of NPs containing free doxorubicine at the time of RFA or after leads to an increased diameter of coagulated tumor tissue (and increased concentration of doxorubicine in the ablated tumor) [68]. The NPs accumulate in the region of ablation both in the treated tumor (as result of an increased leakage) and in the peripheral region with thermal induced inflammation. This is known as the enhanced permeability and retention (EPR) effect [70]. 
The liposomes were the first NPs that have been utilized in combination with RFA. The studies of Ahmed and Goldberg demonstrated that the use of lipid NPs as carriers of a drug combined with ARF was associated with an increased accumulation of doxorubicin in the tumor, while non-encapsulated free doxorubicin did not have increased tumor uptake following RFA [71]. Since then, an important number of investigators improved the lipid layer of liposomes that has contributed to enhanced tumor damage secondary to formation of lipid hydro-peroxide leading to enhanced oxidative stress. Also, the investigators demonstrated that NPs size could influence the intratumoral drug accumulation and tissue coagulation [68].

\subsection{Non-invasive RF nanoablation}

As a negative relationship between the frequency of the waves and the depth of penetration exists, radio waves may be used as an alternative to heat tumors that are deeply located. The heating rate of a certain tissue is described by the formula $\mathrm{HR}=\mathrm{SAR} / 69.77 \mathrm{CH}$ where SAR is the specific absorption rate and $\mathrm{CH}$ the specific heat capacity of the tissue $\left(\mathrm{kcal} / \mathrm{kg}{ }^{\circ} \mathrm{C}\right)$. As SAR $(\mathrm{W} / \mathrm{kg}$ ) depends on the dielectric conductivity of the tissue, an enhanced conductivity provided by AuNPs or carbon nanotubes may increase the heat delivered to the tissue [72].

These low-frequency electromagnetic waves have the advantage to penetrate human tissues and pass through the entire body with minimal perturbations until the RF fields interact with metal. The metal particles absorb RF energy and release heat to the adjacent region. Several reports suggested that tumoral hyperthermia may be improved through the use of targeted nanomaterials, which produce an intracellular hyperthermia and act as RF-thermal transducers, leaving the surrounding healthy tissue intact [68].

The delivery of RF generated heat in deep structures may be achieved either by RF needle inserted into the tumor (standard RFA) or by an external device that generates an RF field $[68,72]$.

If standard RF ablation produces a hyperthermic region of $2-4 \mathrm{~cm}$ diameter around the probe's tip, the nanoparticle-mediated RF field induces a hyperthermic area of approximately $100 \mu \mathrm{m}$. The heating mechanism of NPs in an RF field is a complex phenomenon that is still under debate [73]. Most of the RF field devices produce shortwave RF fields (13.56 MHz), allowing them to be used in the medical field. Several reports have shown that Joule heating of the background ionic suspension where the NPs are suspended can be the main source of RF heat production [74]. A relative high variety of NPs as AuNPs, carbon nanotubes (SWNTs), quantum dots (cadmium-selenide and indium-gallium-phosphide), silicon nanoparticles ( $\mathrm{Si}$ NPs), and La0.7Sr0.3MnO3 (Dex-LSMO) have been associated with RF field [74, 75]. The use of NPs seems to improve the standard RFA by increasing the specificity of tumor destructions and affording a relative target therapy. Between these NPs are several differences, such as the SWNTs are heated faster than AuNPs unlike quantum dots that are heated in a similar manner to AuNPs [73].

SWNTs showed that they can be activated from a distance by RF field to produce thermal cytotoxicity [75]. The SWNTs have been injected in Vx2 tumors and induced the necrosis of all tumors within $5 \mathrm{~min}$ of RF field exposure. Regions of necrosis were identified with 2-5 mm 
borders. It is important to highlight that SWNTs alone or RF field exposure alone did not induce any measurable tumor necrosis or liver injury. In another study, the authors demonstrated that SWNTs injected into malignant cells may allow noninvasive RF field treatments to produce lethal thermal injury to the malignant cells. In a similar study conducted by Raoof, Hep3B and HepG2 cells were injected to kentera modified SWNT and were exposed to an 800 W RF field. Significant thermal cytotoxicity was demonstrated with 2 min of RF exposure in a concentration-dependent manner [75]. Also the group conducted by Cardinal obtained similar results after they exposed a rat model (with HepG2 cells) into an RFA field following the administration of AuNPs [76]. In a study conducted by Glazer ES, AuNPs utilized cetuximabconjugated AuNPs in nonionizing RF radiation to investigate human pancreatic xenograft destruction in a murine model [73]. The result showed an increased apoptosis with decreased viability of tumoral cells after treatment with cetuximab-conjugated AuNPs and RF field exposure. Another important observation was the lack of injury to other organs.

It becomes a reality the fact that nanotechnologies will play a major role in new antitumoral therapies. In the last years, the thermal approach using nanoparticles, nanoemulsion, $\mathrm{pH}$ responsive nanoparticles, nanoparticles combined with radiation, and nanovectors for drug delivery have been the most evaluated nanoparticle-based cancer treatment methods. The ability of SWNTs to convert NIR laser radiation into heat, due to the photon-phonon and electron interactions, provides the opportunity to create a new generation of immunoconjugates for cancer phototherapy. In 2011, Iancu et al. demonstrated that the HepG2 cells treated with multi-walled carbon nanotubes (HSA-MWCNTs) following laser irradiation had a higher necrotic rate compared with normal cells [77].

\subsection{Thermosensitive liposomes currently in advanced clinical trials}

Discovered in 1964 by Alec Bangham, liposomes are self-assembling, biocompatible, biodegradable, and nonimmunogenic nanovesicles consisting of a lipid bilayer enclosing an aqueous phase [78]. The features of liposomes allow for a wide range of drug delivery; consequently, hydrophilic drugs can be trapped in the liposome's aqueous compartments while the lipid bilayer can be utilized to incorporate hydrophobic drugs. Due to the discontinuous endothelial lining and the lack of efficient lymphatic drainage of the tumor, the extravasations of liposomes into the interstitial space is increased and the liposomes can accumulate in the tumoral tissue; therefore, they will function as a sustained drug-release formula [79]. Immordino mentioned for the first time this process and named it as EPR effect [80]. Moreover, the combination (liposome-chemotherapy) changes drug pharmacokinetic properties and minimizes its systemic toxicity. Furthermore, the drug prevents the entrapped drug from premature inactivation in the circulation. The main issue of liposomes is that they are rapidly phagocytized by the mononuclear phagocyte (MP) and removed from the blood circulation after intravenous injection. To avoid this inconvenience, the authors developed a grafting poly(ethylene glycol) (PEG) or oligoglycerol-moieties on the surface of the liposomal carrier. By reducing MP system uptake [80], long-circulating PEGylated liposomes can passively accumulate into solid tumors undergoing angiogenesis. Another improvement was the incorporation of additional lipid compounds that further enhance membrane permeability at the phase 
transition temperature of the lipid membrane (lysolipid or oligoglycerol-polyglycol) [79, 8184]. The result was a long blood circulation time in vivo. These types of low temperature thermosensitive liposomes (LTSLs)[79] are injected just prior to or during the HT treatment, with immediate release of their contents upon arrival in the heated tumor area.

The main limit of this type of therapy remains the intimate relation between the biodisponibility of liposomes and the vascular permeability. It is important to underline that vascular permeability between different tumor types and even within tumors can be highly variable, resulting in unpredictable liposome extravasation into the tumor tissue [85, 86]. Due to the combination of sub-optimal drug release kinetics and unpredictable vascular permeability, only modest results in the therapeutic index of chemotherapy have been obtained using liposomes for target drug delivery [87].

An important progress in the use of liposomes was the invention of small, $100 \mathrm{~nm}$-long circulating liposomes that have a long blood-residence time as their main characteristic. These favorable circulation properties resulted in an enhanced accumulation of liposomal drugs in the tumor area.

To date, several liposomal products have been approved for clinical use: liposomes with doxorubicin (Doxil/Caelyx, Myocet, and Lipo-Dox) for treatment of Kaposi's sarcoma, ovarian cancer, breast cancer, and multiple myeloma; liposomes with daunorubicin (DaunoXome) for treatment of Kaposi's sarcoma; and liposomes encapsulating vincristine (Marqibo) for acute lymphoblastic leukemia [88].

Hyperthermia represents the heating of tumors to temperatures of up to $43^{\circ} \mathrm{C}$. The main effect consists of an increased tissue perfusion, oxygenation and blood flow velocity, and microvessel permeability contributing to increased antibodies, drug, or nanoparticles levels in tumors at clinically tolerated temperatures [89-92]. Nowadays, hyperthermia for triggering TSLs is applied locally and in a noninvasive way from an external source to a targeted area using focused ultrasound technology (FUS) and high-intensity focused ultrasound (HIFU), or invasively using ARF or MWA [93, 94]. For superficial tumors, the authors used regional HT and external antennas or applicators that emit microwaves or radio waves. Localized HT is used to destroy deeply located tumors. The antennas (microwave antennas, radiofrequency electrodes) are inserted directly within the tumor. The major limit of this heating method is the tumor diameter (less than $5 \mathrm{~cm}$ ). Focused ultrasound is used to heat small lesions $(\mathrm{mm})$. In a recent study Dromi et al. combined LTSLs with hyperthermia from FUS [95]. They obtained an increased drug discharge at the tumoral area and the most important tumor had a delayed growth.

The newest heating method is magnetic resonance guided focused ultrasound technology (MRgFUS). These combinations allow simultaneous treatments, imaging to guide the treatment and MR thermometry to noninvasively monitor temperature changes and assure feedback in real-time [87]. In two recent studies, the authors used MRgFUS and drug-loaded liposome in rat [96] and rabbit [97] models. The results showed that the combination MRgFUS with drug loaded liposome assured the greatest uptake of the drug when compared to controls (liposome only and/or free drug). Several studies have analyzed the combination of RFA and 
the non-thermally sensitive liposomal doxorubicin, showing larger ablation zones compared with RFA alone, both at the preclinical and clinical levels. The suggested mechanisms for the synergistic effect of liposomal doxorubicine and RFA are as follows: increased markers of DNA breakage, oxidative stress and apoptosis, increased heat-shock protein 70 in the areas surrounding the ablation zone after combination treatment $[98,99]$. In addition, Ahmed and colleagues observed that after combining RFA with Doxil, the intratumoral drug uptake increased, while the dose of doxorubicin necessary for tumor destruction decreased [100].

In order to optimize the effects of liposomes, the use of TSLs that trigger the release of the drug at the edge of the heated zone was suggested [101-103]. These TSLs contain thermosensitive lipids in their bilayer, undergoing a gel-to-liquid phase transition at the desired temperature (usually between $41^{\circ} \mathrm{C}$ and $43^{\circ} \mathrm{C}$ ), after which the drug enters tumor cells in free form. This conversion is the consequence of a conformational change in the alkyl chains of the lipids, which leads to an increase in the volume occupied by the hydrocarbon chains in the membrane and thus an increase in the permeability of the lipid bilayer [79]. Common TSLs have been composed from 1, 2-dipalimitoyl-sn -glycero-3-phosphocholine (DPPC) as the primary lipid, because its phase transition temperature (Tm) occurs at $41.5^{\circ} \mathrm{C}$.

In 2009, TSLs containing Dox known as ThermoDox®, became the first heat-triggered release formula of the anthracycline doxorubicin that reached pharmaceutical development (Celsion Corporation, Columbia, Maryland, USA) and clinical application [104-105]. Thermodox® is composed of DPPC:MSPC:DSPE-PEG2000 (86:10:4 molar ratio) and in combination with mild was used in the Phase III clinical trial to treat hepatocellular carcinoma and the Phase II trial in combination with local mild for patients with recurrent breast cancer of the chest wall and colorectal liver. After intravenous administration, Thermodox ${ }^{\circledR}$ concentrates in the liver where it rapidly permeates HCC lesions and their vasculature. Regarding safety and tolerability, in Phase I ThermoDox ${ }^{\circledR}$ was associated with low side effects and the maximum tolerated dose was established at $50 \mathrm{mg} / \mathrm{m} 2$. According to the Phase I trial, RFA and ThermoDox ${ }^{\circledR}$ may be used as a front-line therapy for HCC $>3 \mathrm{~cm}$ [106]. Unfortunately, in 2013 Celsion Corp. was unable to demonstrate the effectiveness of ThermoDox® in the improvement of free survival [79]. It seemed that the temperature of drug release is different between in vivo and in vitro. In a study conducted by Hossann, about $90-100 \%$ Dox release from LTSLs in plasma or serum at $39-40^{\circ} \mathrm{C}$ resulted in $2^{\circ} \mathrm{C}$ below the theoretical temperature [107]. Therefore, it might be that all drug content is released from the LTSLs below $41-42^{\circ} \mathrm{C}$, which means that the drug is discharged in blood circulation before the accumulation of LTLS in the target heated tumoral area [79]. In a recent study, after the incorporation of lysophosphatidylcholines (lyso-PC, e.g. 1-stearoyl-2-hydroxy-sn-glycero-3-phosphocholine, MSPC) into the liposomal membrane, it was possible to further accelerate the encapsulated drug at Tm [108].

Fine tunings in drug release kinetics of LTSLs was demanded to assure an improved dug release [109]. In 2014, Chen J evaluated [79] high temperature triggered TSLs (HTSLs) composed of DPPC and hydrogenated soy phosphatidylcholine (HSPC). For these types of liposomes, the theoretical temperature of discharge of HTSLs was set at $44^{\circ} \mathrm{C}$; thus, the body temperature had less influence on the drug release from the vesicles. The result of this study 
was encouraging. Compared to conventional LTSLs, the new formula of HTSLs was associated with higher stability and less content discharge to the heated tumor area.

Several authors recommended the attaching of targeting ligands to the nanoparticles to assure a more specific localization and retention of the liposomal drug in tumors. Another reason to utilize these ligands is the capacity of promoting active cellular uptake of the drug-containing nanoparticles through binding to targeted internalizing receptors [110-112].

The cationic TSLs, called CTSLs (cationic thermosensitive liposome) is a new class of LTSL that contains a cationic lipid in its membrane. The CTSLs are absorbed by vascular endothelium and tumor cells; afterwards, they release their contents upon applying a temperature trigger [113]. It seems that, once accumulated, rapid drug release by intracellular cationic liposomes may achieve high intracellular concentrations of drug, thereby maximizing damage to both the endothelial cell and tumor cell compartments [113]. To evaluate tumoral accumulation of liposomes, radionuclides and nuclear imaging may be used. Even if the authors have obtained good results, in the future these types of treatment will have to demonstrate their therapeutic potential in clinical practice.

\section{Conclusions and future perspectives}

As we have already seen, there are several types of thermal-based therapies that have shown modest efficacy in HCC treatment. Unfortunately, simple heating techniques have trouble discriminating between tumors and surrounding healthy tissues. Moreover, the use of thermal therapies in large HCC is of limited value. In order to overcome these limitations many groups have investigated the use of NPs to increase the tumor ablation zone.

There are many types of NPs, each type with its own major advantages and disadvantages. Based on currently available literature, we could not say which of the above-described NPs is better for the long-term management of HCC. Unfortunately, there are no studies comparing AuNPs with carbon nanoparticles or magnetic nanoparticle. The use of NPs such as AuNPs, carbon nanoparticles, and magnetic nanoparticles have shown great promise as light absorbers for cancer therapy, demonstrating an ability to destroy cancerous lesions both in vivo and in vitro [31].

We believe that an ideal NP should be a good light absorber in order to achieve complete ablation of the tumor tissues. To avoid systemic toxicity, the NPs should show selective accumulation in target tissue with minimal nonspecific distribution. Not at least, they should be rapidly cleared from the body after their mission to prevent redistribution into off-target sites [38].

Future research should focus on the development of multifunctional NP. For instance, theranostic agents could improve both the diagnostic accuracy and therapy of HCC. Small HCC means better outcomes. The majority of NPs are functionalized to target the tumor cells, leaving the tumor stroma unaffected. A pro-tumorigenic stroma or better said a pro-tumori- 
genic microenvironment could lead to tumor recurrence, therefore dual targeting of both tumor cells and tumor stroma could overcome these limitations.

Specific targeting in HCC is still a major problem. There are many molecular pathways involved in HCC development. Moreover, not all HCC express the same receptors on the cell surface. In order to specifically deliver NP in the tumor area, immunohistological staining must be performed. This is hard to perform, particularly in HCC, since liver biopsy is no longer recommended for HCC diagnosis. Maybe it is time to go back where we started and reconsider the role of liver biopsy in HCC management.

In the last 50 years, despite tremendous advances in our knowledge of the molecular mechanism of cancer, there has been no change in the age-adjusted mortality from cancer [39]. This data clearly suggests that what we are doing now is wrong and an individualized treatment could bring new hopes for HCC patients.

\section{Acknowledgements}

This material was financed by the partnership program in priority areas - PN II, implemented with support from the National Authority of Scientific Research (ANCS), CNDI - UEFISCDI, project nr. 2011-3.1-0252 (NANO- ABLATION).

\section{Author details}

Zeno Sparchez ${ }^{1 *}$, Tudor Mocan ${ }^{2}$ and Pompilia Radu ${ }^{2}$

*Address all correspondence to: zsparchez@yahoo.co.uk

1 Iuliu Hatieganu University of Medicine and Pharmacy, Cluj Napoca, Romania

2 Institute for Gastroenterology and Hepatology, Cluj Napoca, Romania

\section{References}

[1] Kim YS, Lim HK, Rhim H, Lee MW. Ablation of hepatocellular carcinoma. Best Pract Res Clin Gastroenterol. 2014;28(5):897-908.

[2] Gillams AR. Liver ablation therapy. Br J Radiol. 2004;77(92):713-723.

[3] Shiina S, Tateisshi R, Imamura M, Teratani T, Koike Y, Sato S, et al. Percutaneous ethanol injection for hepatocellular carcinoma: 20-year outcome and prognostic factors. Liver Int. 2012;32(9):1434-1442. 
[4] McWilliams JP, Yamamoto S, Raman SS, Loh CT, Lee EW, Liu DM, et al. Percutaneous ablation of hepatocellular carcinoma: current status. J Vasc Interv Radiol. 2010;21(8 Suppl):S204-213.

[5] Lin SM, Lin CJ, Lin CC, Hsu CW, Chen YC. Randomised controlled trial comparing percutaneous radiofrequency thermal ablation, percutaneous ethanol injection, and percutaneous acetic acid injection to treat hepatocellular carcinoma of $3 \mathrm{~cm}$ or less. Gut. 2005;54(8):1151-1156.

[6] Chen MS, Li JQ, Zheng Y, Guo RP, Liang HH, Zhang YQ, et al. A prospective randomized trial comparing percutaneous local ablative therapy and partial hepatectomy for small hepatocellular carcinoma. Ann Surg. 2006;243(3):321-328.

[7] Brunello F, Veltri A, Carucci P, Pagano E, Ciccone G, Moretto P, et al. Radiofrequency ablation versus ethanol injection for early hepatocellular carcinoma: aA randomized controlled trial. Scand J Gastroenterol. 2008;43(6):727-735.

[8] Seror O. Percutaneous hepatic ablation: what needs to be known in 2014. Diagn Interv Imaging. 2014;95(7-8):665-675.

[9] Skinner MG, Iizuka MN, Kolios MC, Sherar MD. A theoretical comparison of energy sources-microwave, ultrasound and laser-for interstitial thermal therapy. Phys Med Biol. 1998;43(12):3535-3547.

[10] Yu H, Burke CT. Comparison of percutaneous ablation technologies in the treatment of malignant liver tumors. Semin Intervent Radiol. 2014;31(2):129-137.

[11] Shibata T, Iimuro Y, Yamamoto Y, Maetani Y, Ametani F, Itoh K, et al. Small hepatocellular carcinoma: cComparison of radio-frequency ablation and percutaneous microwave coagulation therapy. Radiology. 2002;223(2):331-337.

[12] Zhang L, Wang N, Shen Q, Cheng W, Qian GJ. Therapeutic efficacy of percutaneous radiofrequency ablation versus microwave ablation for hepatocellular carcinoma. PLoS One. 2013;8(10):e76119.

[13] Christophi C, Muralidharan V. Treatment of hepatocellular carcinoma by percutaneous laser hyperthermia. 2001;16:548-552.

[14] Rapaccini GL. Percutaneous ablative treatments of hepatocellular carcinoma. Hepatology. 2014. doi: 10.1002/hep.27615.

[15] Ferrari FS, Megliola A, Scorzelli A, Stella A, Vigni F, Drudi FM, et al. Treatment of small HCC through radiofrequency ablation and laser ablation. Comparison of techniques and long-term results. Radiol Med. 2007;112(3):377-393.

[16] Di Costanzo GG, Tortora R, D'Adamo G, De Luca M, Lampasi F, Addario L, et al. Radiofrequency ablation versus laser ablation for the treatment of small hepatocellular carcinoma in cirrhosis: A randomized trial. J Gastroenterol Hepatol. 2015;30(3): 559-565. 
[17] Maccini M, Sehrt D, Pompeo A, Chicoli FA, Molina WR, Kim FJ. Biophysiologic considerations in cryoablation: aA practical mechanistic molecular review. Int Braz J Urol. 2011;37(6):693-696.

[18] Wang C, Wang H, Yang W, Hu K, Xie H, Hu KQ, et al. A multicenter randomized controlled trial of percutaneous cryoablation versus radiofrequency ablation in hepatocellular carcinoma. Hepatology. 2014. doi: 10.1002/hep.27548.

[19] Lee EW, Thai S, Kee ST. Irreversible electroporation: A novel image-guided cancer therapy. Gut Liver. 2010;4(Suppl 1):S99-S104.

[20] Scheffer HJ, Nielsen K, de Jong MC, van Tilborg AA, Vieveen JM, Bouwman AR, et al. Irreversible electroporation for nonthermal tumor ablation in the clinical setting: aA systematic review of safety and efficacy. J Vasc Interv Radiol. 2014;25(7):997-1011.

[21] Bardhan R1, Lal S, Joshi A, Halas NJ. Theranostic nanoshells: fFrom probe design to imaging and treatment of cancer. Acc Chem Res. 2011;44(10):936-946.

[22] Hwang S, Nam J, Jung S, Song J, Doh H, Kim S. Gold nanoparticle-mediated photothermal therapy: Current status and future perspective. Nanomedicine (Lond). 2014;9(13):2003-2022.

[23] Kennedy LC, Bickford LR, Lewinski NA, Coughlin AJ, Hu Y, Day ES, et al. A new era for cancer treatment: gGold-nanoparticle-mediated thermal therapies. Small. 2011;7(2):169-183.

[24] Liu SY, Liang ZS, Gao F, Luo SF, Lu GQ. In vitro photothermal study of gold nanoshells functionalized with small targeting peptides to liver cancer cells. J Mater Sci Mater Med. 2010;21(2):665-74.

[25] You J, Zhang R, Xiong C, Zhong M, Melancon M, Gupta S, et al. Effective photothermal chemotherapy using doxorubicin-loaded gold nanospheres that target EphB4 receptors in tumors. Cancer Res. 2012;72(18):4777-4786.

[26] Gupta S, Stafford RJ, Javadi S, Ozkan E, Ensor JE, Wright KC, et al. Effects of nearinfrared laser irradiation of biodegradable microspheres containing hollow gold nanospheres and paclitaxel administered intraarterially in a rabbit liver tumor model. J Vasc Interv Radiol. 2012;23(4):553-561.

[27] Zhenglin Li, Yongyi Zeng, Da Zhang, Ming Wu, Lingjie Wu, Aimin Huang, et al. Glypican-3 antibody functionalized Prussian blue nanoparticles for targeted MR imaging and photothermal therapy of hepatocellular carcinoma. J. Mater. Chem. B. 2014;2:3686-3696.

[28] Zhao J, Vykoukal J, Abdelsalam M, Recio-Boiles A, Huang Q, Qiao Y, et al. Stem cellmediated delivery of SPIO-loaded gold nanoparticles for the theranosis of liver injury and hepatocellular carcinoma. Nanotechnology. 2014;25(40):405101. doi: 10.1088/0957-4484/25/40/405101. 
[29] Singh R, Torti SV. Carbon nanotubes in hyperthermia therapy. Adv Drug Deliv Rev. 2013;65(15):2045-20460.

[30] Robinson JT, Welsher K, Tabakman SM, Sherlock SP, Wang H, Luong R, et al. High performance in vivo near-IR $(>1 \mu \mathrm{m})$ imaging and photothermal cancer therapy with carbon nanotubes. Nano Res. 2010;3(11):779-793.

[31] Manthe RL, Foy SP, Krishnamurthy N, Sharma B, Labhasetwar V. Tumor ablation and nanotechnology. Mol Pharm. 2010;7(6):1880-1898.

[32] Asharani PV, Hande MP, Valiyaveettil S. Anti-proliferative activity of silver nanoparticles. BMC Cell Biol. 2009;10:65.

[33] Mironava T, Hadjiargyrou M, Simon M, Jurukovski V, Rafailovich MH. Gold nanoparticles cellular toxicity and recovery: eEffect of size, concentration and exposure time. Nanotoxicology. 2010;4(1):120-137.

[34] Kang B, Mackey MA, El-Sayed MA. Nuclear targeting of gold nanoparticles in cancer cells induces DNA damage, causing cytokinesis arrest and apoptosis. J Am Chem Soc. 2010;132(5):1517-1519.

[35] Ziyad S, Iruela-Arispe ML. Molecular mechanisms of tumor angiogenesis. Genes Cancer. 2011;2(12):1085-1096.

[36] Danhier F, Vroman B, Lecouturier N, Crokart N, Pourcelle V, Freichels H, et al. Targeting of tumor endothelium by RGD-grafted PLGA-nanoparticles loaded with paclitaxel. J Control Release. 2009;140(2):166-173.

[37] Lunov O, Syrovets T, Büchele B, Jiang X, Röcker C, Tron K, et al. The effect of carboxydextran-coated superparamagnetic iron oxide nanoparticles on c-Jun N-terminal kinase-mediated apoptosis in human macrophages. Biomaterials. 2010;31(19): 5063-50671.

[38] Kobayashi T. Biotechnol J. Cancer hyperthermia using magnetic nanoparticles. 2011;6(11):1342-1347.

[39] Tartaj P, Puerto Morales P, Veintemillas-Verdaguer S, Carre T, Carlos J Serna J. The preparation of magnetic nanoparticles for applications in biomedicine Phys. D: Appl. Phys. 2003;36:182-197

[40] Batlle X, Labarta A. Finite-size effects in fine particles: mMagnetic and transport properties J. Phys. D: Apply. Phys. 2002;35:R15.

[41] Johannsen M, Gneveckow U, Thiesen B, Taymoorian K, Cho CH, Waldöfner N, et al. Thermotherapy of prostate cancer using magnetic nanoparticles: fFeasibility, imaging, and three dimensional temperature distribution. Eur Urol 2007;52(6):1653-1661.

[42] Rosensweig RE. Heating magnetic fluid with alternating magnetic field. J Magn Magn Mater. 2002;252(1-3):370-374. 
[43] Huang HS, Hainfeld JF. Intravenous magnetic nanoparticle cancer hyperthermia. Int J Nanomedicine. 2013;8:2521-253.

[44] Jordan A, Scholz R, Wust P, Schirra H, Thomas Schiestel, Schmidt H, Felix R. Endocytosis of dextran and silan-coated magnetite nanoparticles and the effect of intracellular hyperthermia on human mammary carcinoma cells in vitro. Journal of Magnetism and Magnetic Materials 1999;194:185-196.

[45] Moroz P, Jones SK, Gray BN. Magnetically mediated hyperthermia: Current status and future directions. Int J Hyperthermia. 2002;18(4):267-284.

[46] Srivastava PK1, Menoret A, Basu S, Binder RJ, McQuade KL. Heat shock proteins come of age: Primitive functions acquired new roles in an adaptive world. Immunity. 1998;8(6):657-665.

[47] Jordan A, Scholz R, Maier-Hauff K, Johannsen M, Wust P, Nadobny J, et al. J. Magn. Magn. Mater. 2001;225.

[48] Hilger I, Frühauf K, Andrä W, Hiergeist R, Hergt R, Kaiser WA. Heating potential of iron oxides for therapeutic purposes in interventional radiology. Acad Radiol. 2002;9(2):198-202.

[49] Hyeon T. Chemical synthesis of magnetic nanoparticles. Chem Commun. 2003;8:927-934.

[50] Gilchrist RK, Medal R, Shorey WD, Hanselman RC, Parrott JC, Taylor CB. Selective inductive heating of lymph nodes. Annals of surgery. 1957;146(4):596-606.

[51] Moroz P, Jones SK, Winter J, Gray BN. Targeting liver tumors with hyperthermia: fFerromagnetic embolization in a rabbit liver tumor model. J Surg Oncol 2001;78(1): 22-29.

[52] Jimbow K, Takada T, Sato M, Sato A, et al. Melanin biology and translational research strategy; melanogenesis and nanomedicine as the basis for melanoma-targeted DDS and chemothermoimmunotherapy. Pigm. Cell Melanoma Res. 2008:21:243-244.

[53] Jordan A, Scholz R, Maier-Hauff K, van Landeghem FK, Waldoefner N, Teichgraeber $\mathrm{U}$, et al. The effect of thermotherapy using magnetic nanoparticles on rat malignant glioma. J Neurooncol. 2006;78(1):7-14

[54] Johannsen M, Jordan A, Scholz R, Koch M, Lein M, Deger S, et al. Evaluation of magnetic fluid hyperthermia in a standard rat model of prostate cancer. J Endourol. 2004;18 (5):495-500.

[55] Johannsen M, Thiesen B, Jordan A, Taymoorian K, Gneveckow U, Waldöfner N, et al. Magnetic fluid hyperthermia (MFH) reduces prostate cancer growth in the orthotopic Dunning R3327 rat model. Prostate. 2005;64:283-292. 
[56] Johannsen M, Thiesen B, Gneveckow U, Taymoorian K, Waldöfner N, Scholz R, et al. Thermotherapy using magnetic nanoparticles combined with external radiation in an orthotopic rat model of prostate cancer. Prostate. 2006;66:97-104.

[57] Shinkai M, Yanase M, Honda H, Wakabayashi T, Yoshida J, Kobayashi T. Intracellular hyperthermia for cancer using magnetite cationic liposome-in vitro study. Jpn. J. Cancer Res. 1996:87;1179.-183.

[58] Ito A, Tanaka K, Honda $\mathrm{H}$, Abe $\mathrm{S}$ et al., Complete regression of mouse mammary carcinoma with a size greater than $15 \mathrm{~mm}$ by frequent repeated hyperthermia using magnetite nanoparticles. J. Biosci. Bioeng. 2003;96:364-369.

[59] Zhang J, Misra RD. Magnetic drug-targeting carrier encapsulated with thermosensitive smart polymer: core-shell nanoparticle carrier and drug release response. Acta Biomater. 2007;3(6):838-850.

[60] Hu SH, Liao BJ, Chiang CS, Chen PJ, Chen IW, Chen SY. Core-shell nanocapsules stabilized by single-component polymer and nanoparticles for magneto-chemotherapy/ hyperthermia with multiple drugs. Adv Mater. 2012;24(27):3627-3632.

[61] Johannsen M, Gneveckow U, Taymoorian K, Cho CH, Thiesen B, Scholz R, et al. Thermal therapy of prostate cancer using magnetic nanoparticles. Actas Urol Esp. 2007;31(6):660-667.

[62] Maier-Hauff K, Rothe R, Scholz R, Gneveckow U, Wust P, Thiesen B, et al. Intracranial thermotherapy using magnetic nanoparticles combined with external beam radiotherapy: rResults of a feasibility study on patients with glioblastoma multiforme. J Neurooncol. 2007;81(1):53-60.

[63] van Landeghem FK, Maier-Hauff K, Jordan A, Hoffmann KT, Gneveckow U, Scholz $\mathrm{R}$, et al. Post-mortem studies in glioblastoma patients treated with thermotherapy using magnetic nanoparticles. Biomaterials. 2009;30(1):52-57.

[64] Takamatsu S, Matsui O, Gabata T, Kobayashi S, Okuda M, Ougi T, et al. Selective induction hyperthermia following transcatheter arterial embolization with a mixture of nano-sized magnetic particles (ferucarbotran) and embolic materials: Feasibility study in rabbits. Radiat Med. 2008;26:179-187.

[65] Yuan $Y$, Wyatt C, Maccarini P, et al. A heterogeneous human tissue mimicking phantom for RF heating and MRI thermal monitoring verification. Phys Med Biol. 2012;57(7):2021-2037.

[66] Wiekhorst F, Steinhoff U, Eberbeck D, Trahms L. Magnetorelaxometry assisting biomedical applications of magnetic nanoparticles. Pharm Res. 2012;29(5):1189-1202.

[67] Widmann G, Schullian P, Haidu M, Bale R. Stereotactic radiofrequency ablation (SRFA) of liver lesions: tTechnique effectiveness, safety, and interoperator performance. Cardiovasc Intervent Radiol. 2012;35(3):570-580. 
[68] Phillips WT, Bao A, Brenner AJ, Goins BA. Image-guided interventional therapy for cancer with radiotherapeutic nanoparticles. Adv Drug Deliv Rev. 2014, 30;76: 39-59.

[69] Shenoi MM, Anderson J, Bischof JC. Nanoparticle enhanced thermal therapies. Conf Proc IEEE Eng Med Biol Soc. 2009;2009:1979-1982.

[70] Torchilin V. Tumor delivery of macromolecular drugs based on the EPR effect. Adv. Drug Deliv. Rev. 2011;63:131-135.

[71] Ahmed M, Goldberg SN. Combination radiofrequency thermal ablation and adjuvant IV liposomal doxorubicin increases tissue coagulation and intratumoural drug accumulation. Int J Hyperthermia. 2004;20(7):781-802.

[72] Day ES, Morton JG, West JL. Nanoparticles for thermal cancer therapy. J Biomech Eng. 2009;131(7):074001.

[73] Glazer ES, Curley SA. Non-invasive radiofrequency ablation of malignancies mediated by quantum dots, gold nanoparticles and carbon nanotubes. Ther Deliv. 2011;2:1325-1330.

[74] Corr SJ, Cisneros BT, Green L, Raoof M, Curley SA. Protocols for assessing radiofrequency interactions with gold nanoparticles and biological systems for non-invasive hyperthermia cancer therapy. J Vis Exp. 2013;28:(78).

[75] Raoof M, Curley SA. Non-invasive radiofrequency-induced targeted hyperthermia for the treatment of hepatocellular carcinoma. Int J Hepatol. 2011;676957. doi: $10.4061 / 2011 / 676957$.

[76] Cardinal J, Klune JR, Chory E, Jeyabalan G, Kanzius JS, Nalesnik M, Geller DA. Noninvasive radiofrequency ablation of cancer targeted by gold nanoparticles. Surgery. 2008;144(2):125-132.

[77] Iancu C, Mocan L, Bele C, Orza AI, Tabaran FA, Catoi C, Stiufiuc R, Stir A, Matea C, Iancu D, Agoston-Coldea L, Zaharie F, Mocan T. Enhanced laser thermal ablation for the in vitro treatment of liver cancer by specific delivery of multiwalled carbon nanotubes functionalized with human serum albumin. Int $\mathrm{J}$ Nanomedicine. 2011;6:129-141.

[78] Bangham AD, Horne RW. Negative staining of phospholipids and their structural modification by surface-active agents as observed in the electron microscope. J. Mol. Biol. 1964;8:660-668.

[79] Chen J, He CQ, Lin AH, Gu W, Chen ZP, Li W, Cai BC. Thermosensitive liposomes with higher phase transition temperature for targeted drug delivery to tumor. Int $\mathrm{J}$ Pharm. 2014;475(1-2):408-415.

[80] Immordino ML, Dosico F, Cattel L. Stealth liposomes: review of the basic science, rationale, and clinical applications:, existing and potential. Int. J. Nanomed. 2006;1:297-315. 
[81] Needham D, Anyarambhatla G, Kong G, Dewhirst MW. A new temperature sensitive liposome for use with mild hyperthermia: cCharacterization and testing in a human tumor xenograft model. Cancer Res. 2000;60(5):1197-1201.

[82] Unezaki S, Maruyama K, Takahashi N, Koyama M, Yuda T, Suginaka A, Iwatsuru M. Enhanced delivery and antitumor activity of doxorubicin using long-circulating thermosensitive liposomes containing amphipathic polyethylene glycol in combination with local hyperthermia. Pharm Res. 1994;11(8):1180-1185.

[83] Lindner LH, Eichhorn ME, Eibl H, Teichert N, Schmitt-Sody M, Issels RD, Dellian M. Novel temperature-sensitive liposomes with prolonged circulation time. Clin Cancer Res. 2004;10(6):2168-2178.

[84] Li T, Zhang M, Han Y, Zhang H, Xu L, Xiang Y. Targeting therapy of choroidal neovascularization by use of polypeptide- and PEDF-loaded immunoliposomes under ultrasound exposure. J Huazhong Univ Sci Technolog Med Sci. 2010;30(6):798-803.

[85] Seynhaeve AL, Hoving S, Schipper D, Vermeulen CE, de Wiel-AmbagtsheerGa, van Tiel ST, et al. Tumor necrosis factor alpha mediates homogeneous distribution of liposomes in murine melanoma that contributes to a better tumor response. Cancer Res. 2007;67(19):9455-9462.

[86] Harrington KJ, Mohammadtaghi S, Uster PS, Glass D, Peters AM, Vile RG, Stewart JS. Effective targeting of solid tumors in patients with locally advanced cancers by radiolabeled pegylated liposomes. Clin Cancer Res. 2001;7(2):243-254.

[87] Ta T, Porter TM. Thermosensitive liposomes for localized delivery and triggered release of chemotherapy. J ControlRelease. 2013;10 169(1-2):112-125.

[88] Allen TM, Cullis PR. Liposomal drug delivery systems: From concept to clinical applications. Adv Drug Deliv Rev. 2013;65:36-48.

[89] Horsman MR, Overgaard J. Can mild hyperthermia improve tumour oxygenation? Int J Hyperthermia. 1997;13(2):141-147.

[90] Karino T, Koga S, Maeta M. Experimental studies of the effects of local hyperthermia on blood flow, oxygen pressure and pH in tumors. Jpn J Surg. 1988;18(3):276-283.

[91] Fujiwara K, Watanabe T. Effects of hyperthermia, radiotherapy and thermoradiotherapy on tumor microvascular permeability. ActaPathol Jpn. 1990;40(2):79-84.

[92] Li L, ten Hagen TL, Bolkestein M, Gasselhuber A, Yatvin J, van Rhoon GC, Eggermont AM, Haemmerich D, Koning GA. Improved intratumoral nanoparticle extravasation and penetration by mild hyperthermia. J ControlRelease. 2013;28 167(2): 130-137.

[93] Kennedy JE. High-intensity focused ultrasound in the treatment of solid tumours. Nat Rev Cancer. 2005;5:321-392. 
[94] Clement GT. Perspectives in clinical uses of high-intensity focused ultrasound. Ultrasonics. 2004;42:1087-1093.

[95] Dromi S, Frenkel V, Luk A, Traughber B, Angstadt M, Bur M, et al. Pulsed-high intensity focused ultrasound and low temperature-sensitive liposomes for enhanced targeted drug delivery and antitumor effect. Clin Cancer Res. 2007;13(9):2722-2727.

[96] de Smet M, Heijman E, Langereis S, Hijnen NM, Grüll H. Magnetic resonance imaging of high intensity focused ultrasound mediated drug delivery from temperaturesensitive liposomes: an in vivo proof-of-concept study. J. Control. Release 2011;150(1):102-110.

[97] Ranjan A, Jacobs GC, Woods DL, Negussie AH, Partanen A, Yarmolenko PS, et al. Image-guided drug delivery with magnetic resonance guided high intensity focused ultrasound and temperature sensitive liposomes in a rabbit Vx2 tumor model. J. Control. Release 2012;158:487-494.

[98] Ahmed M, Moussa M, Goldberg SN. Synergy in cancer treatment between liposomal chemotherapeutics and thermal ablation. Chem Phys Lipids. 2012;165(4):424-437.

[99] Solazzo SA, Ahmed M, Schor-Bardach R, Yang W, Girnun GD, Rahmanuddin S, Levchenko T, Signoretti S, Spitz DR, Torchilin V, Goldberg SN. Liposomal doxorubicin increases radiofrequency ablation-induced tumor destruction by increasing cellular oxidative and nitrative stress and accelerating apoptotic pathways. Radiology. 2010;255(1):62-67.

[100] Ahmed M, Monsky WE, Girnun G, Lukyanov A, D'Ippolito G, Kruskal JB, Stuart KE, Torchilin VP, Goldberg SN. Radiofrequency thermal ablation sharply increases intratumoral liposomal doxorubicin accumulation and tumor coagulation. Cancer Res. 2003;63(19):6327-6333.

[101] Yatvin MB, Weinstein JN, Dennis WH, Blumenthal R. Design of liposomes for enhanced local release of drug by hyperthermia. Science. 1998;202:1290-129.

[102] Mills JK, Needham D. The materials engineering of temperature-sensitive liposomes. Methods Enzymol. 2004;387:82-113.

[103] Needham D, Anyarambhatla G, Kong G, Dewhirst MW. A new temperature sensitive liposome for use with mild hyperthermia: cCharacterization and testing in a human tumor xenograft model. Cancer Res. 2000;60(5):1197-1201.

[104] Poon RTP, Borys N. Lyso-thermosensitive liposomal doxorubicin: aA novel approach to enhance efficacy of thermal ablation of liver cancer. Expert Opin Pharmacother. 2009:10;333-342.

[105] Landon CD, Park J, Needham D, Dewhirst MW. Nanoscale drug delivery and hyperthermia: tThe materials designand preclinical and clinical testing of low temperaturesensitive liposomes used in combination with mild hyperthermia in the treatment of local cancer. Open Nanomed J. 2011;3:38-64. 
[106] Poon RT, Borys N. Lyso-thermosensitive liposomal doxorubicin: An adjuvant to increase the cure rate of radiofrequency ablation in liver cancer. Future Oncol. 2011;7(8):937-945.

[107] Hossann M, Syunyaeva Z, Schmidt R, Zengerle A, Eibl H, Issels RD, Linder, LH. Proteins and cholesterol lipid vesicles are mediators of drug release from thermosensitive liposomes. J Control. Release 2012:162;400-406.

[108] Mills J.K, Needham D. Lysolipid incorporation in dipalmitoylphosphati-dylcholine bilayer membranes enhances the ion permeability and drug release rates at the membrane phase transition. Biochim Biophys Acta. 2005:1716;77-96.

[109] Park K. Lessons learned from thermosensitive liposomes from improved chemotherapy. J Control.Release 2014;174:219.

[110] Park JW, Hong K, Carter P, Asgari H, Guo LY, Keller GA, Wirth C, Shalaby R, Kotts C, Wood WI, et al.. Development of anti-p185HER2 immunoliposomes for cancer therapy. Proc Natl Acad Sci USA. 1995;92(5):1327-1331.

[111] Kullberg M, Mann K, Owens JL. A two component drug delivery system using Her-2-targeting thermosensitive liposomes. J Drug Target. 2009;17(2):98-107.

[112] ] Negussie AH, Miller JL, Reddy G, Drake SK, Wood BJ, Dreher MR. Synthesis and in vitro evaluation of cyclic NGR peptide targeted thermally sensitive liposome. J Control. Release 2010;143(2): 265-273.

[113] Dicheva BM, ten Hagen TL, Li L, Schipper D, Seynhaeve AL, van Rhoon GC, Eggermont AM, Lindner LH, Koning GA. Cationic thermosensitive liposomes: aA novel dual targeted heat-triggered drug delivery approach for endothelial and tumor Cells. Nano Lett. 2013;13(6):2324-2331. 